Article

\title{
Estimating Destination of Bus Trips Considering Trip Type Characteristics
}

\author{
Soongbong Lee ${ }^{1}$, Jongwoo Lee ${ }^{2}$, Bumjoon Bae ${ }^{3}$, Daisik Nam ${ }^{4}$ and Seunghoon Cheon ${ }^{5, *}$ \\ 1 Big Data Platform and Data Economy, The Korea Transport Institute 370 Sicheong-daero, Sejong, 30147, \\ Republic of Korea; habanera82@koti.re.kr \\ 2 Big Data Platform and Data Economy, The Korea Transport Institute 370 Sicheong-daero, Sejong, 30147, \\ Republic of Korea; jongwoo0214@koti.re.kr \\ 3 Center for Privately-Financed Highway Studies, The Korea Transport Institute 370 Sicheong-daero, Sejong, \\ 30147, Republic of Korea; bbae2016@koti.re.kr \\ 4 Graduate School of Logistics, Inha University, Incheon 22212, Korea; namd@inha.ac.kr \\ 5 Big Data Platform and Data Economy, The Korea Transport Institute 370 Sicheong-daero, Sejong, 30147, \\ Republic of Korea; sh1000@koti.re.kr \\ * Correspondence: sh1000@koti.re.kr
}

\begin{abstract}
In recent years, local governments have been using transportation card data to monitor the use of public transport and improve the service. However, local governments that are applying a single-fare scheme are experiencing difficulties in using data for accurate identification of real travel patterns, policy decision support, etc. because the information on alighting stops of users is missing. This policy limits its functionality of utilizing data such as accurate identification of real travel patterns, policy decision support, etc. Various studies to overcome this limitation have been conducted in South Korea and other countries to develop estimation methodologies of alighting stops. Even existing studies introduce an advanced method, we found the margin for better accuracy by combining various estimation methodologies for estimating alighting stops. This study reviewed previously conducted studies to classify data with missing alighting stop information into trip types and then applied an appropriate alighting stop estimation methodology for the characteristics of each trip type by stage. The proposed method is evaluated by utilizing transportation card data of the Seoul metropolitan area and checked the accuracy for each standard of allowable error for sensitivity analysis. Furthermore, the number of trips, accuracy, and valid tag rate were checked for each type to examine the need for classifying the trip types. Finally, our evaluation also examines the impact of classifying trip types on estimation accuracy. The evaluation criteria are accuracy of the number of trips and valid tag rate. The analysis shows that the stage-by-stage estimation methodology based on the trip type proposed in this study can estimate users' destinations more accurately than previous studies. Furthermore, based on the construction of nearly $100 \%$ valid tag data, this study differs from prior studies.
\end{abstract}

Keywords: public transit transaction data; estimation of destination operation information; categorization of trip types; trip chain; travel pattern; historical travel data

\section{Introduction}

Recently, local governments have been using big data in various ways to improve the efficiency and service of transportation operations. In the case of public transportation especially, it is possible to analyze the usage status of public transportation based on transportation card data because most users use transportation cards. In fact, they are used in various areas such as the adjustment of public transport routes and headway of vehicles, usage checking and fare payment, and establishment of a plan for adopting new demandresponsive transport (DRT). 
For effective monitoring of the public transit operation and improvement of the service using transportation card data, it is imperative to identify the travel patterns accurately, including the travel origin and destination of the users. In many cases, however, users do not tag the transportation card on the terminal because many local governments are applying a single-fare scheme. Hence, the information about the alighting stops is missing, and there are difficulties in using the data, such as identifying the actual travel patterns of the users, and monitoring the status and improving the service based on data.

To overcome these limitations, various studies have been conducted in South Korea and other countries to estimate the alighting stops when the alighting tag information of users is missing. In particular, some studies have been conducted focusing on alighting stop estimation methodologies based on the connectivity between trips, i.e., under the assumption that the next trip starts around the destination of this trip [1-18]. Furthermore, studies have been conducted on alighting stop estimation methods by applying machine learning methods and analysis of travel patterns based on the past travel history data [1216]. However, relatively few studies have been conducted on the estimation of more complete travel pattern information in public transit systems by combining various alighting stop estimation methodologies.

In this study, therefore, we estimated real public transport-based travel patterns more accurately and completely by comprehensively considering the characteristics of various alighting stop estimation methodologies and the characteristics of the trips for which input data, alighting tag information is missing; this was to contribute to more effective public transport operation monitoring and policy decision-support of local governments. To this end, we reviewed various alighting stop estimation methods found in previous studies. We considered the characteristics of each method to define the types of travel with missing alighting stop information and set the stages for the alighting stop estimation. The accuracy of the alighting stop information estimation methodology was verified for the Seoul metropolitan area (the alighting tag rate was 98\% as of April 2020), and the accuracy and the valid data ratio were analyzed based on the results estimated after completely deleting the alighting stop information. Furthermore, we examined the accuracy of the stage-by-stage estimation methodology according to the trip type classification and the accuracy of the estimated alighting stops for each standard of allowable error and derived implications based on this.

\section{Literature Review}

\subsection{Methodology for Estimating destination based on trip chain}

A trip-chain-based estimation methodology has been used as the most basic method of estimating alighting stops when alighting tag information is missing. Basically, tripchain-based alighting stop estimation studies assume that the passenger travels many times consecutively and starts the next trip around the destination of the previous trip. Based on this assumption, they estimate alighting stops when there are two or more boarding records per day for the same card ID [1-5]. Some studies assume that the passenger returns to the resident in the case of the last trip on the analyzed day, while the origin of the first trip on a pertinent day is the resident [7-11]. In some other cases, studies look at the origin of the first trip on the next day to estimate the alighting stop of the final trip on that day [12-13]. Studies have also been conducted for a trip-chain-based alighting stop estimation methodology by linking railways and buses [14-15].

Some studies validated the estimation results to improve the success rate and accuracy of alighting stop estimations and analyzed major parameters and sensitivity. Specifically, studies were conducted on the validation of trip-chain-based alighting stop estimation results [16-17]; sensitivity analysis for "allowed walking distance" to determine the continuity between two trips as a major parameter when estimating the alighting stop based on the trip-chain [9-10]; determining whether the passenger transferred and analyzing the walking journey time in terms of the trip-chain-based estimation $[11,18]$. 
The trip-chain-based alighting stop estimation method is applicable only when the trip-chain can be constructed, and it has the limitation that it cannot be applied to a single trip.

\subsection{Methodology for estimating destination based on pattern}

Studies on various techniques, including artificial intelligence (AI) and probability models, have been conducted to estimate the alighting stops for trips with missing alighting stop information when the trip-chain-based alighting estimation is impossible due to the lack of information on the next trip. In the case of analysis-based studies for the past travel history, a study analyzed the distribution of the boarding time of the trip for each passenger and estimated the alighting stops by assuming that the usual boarding stop in the same time band as the estimation-target trip was the origin, while the usual boarding stop in a different time band was the destination [19]; another study analyzed the past travel history of the same card ID to estimate the alighting stop based on the similarity to the estimation-target trip [20]. Moreover, a study estimated alighting stops by applying a three-dimensional latent Dirichlet allocation model [21], and another study analyzed major trip origins, destinations, and transfer locations by spatially clustering the locations of the stops and estimated alighting stops [20]. On the other hand, some studies estimated alighting stops by training models based on various parameters of trips [23-25].

\subsection{Methodology for estimating destination by stage}

If a single estimation methodology, such as a trip-chain-based or AI/probability model-based alighting stop estimation methodology, is applied, there is the limitation that there are trips that are impossible to estimate using the applied methodology. To overcome this limitation, studies have been conducted by applying several alighting stop estimation methods. For example, a study applied the trip-chain-based estimation methodology first and then applied a boarding time similarity-based alighting stop estimation method [19]; a study applied different alighting stop estimation methods by classifying the trip type based on the number of trips per day, the last trip of the day, the transfers in the trip, etc. [26]; a study derived a higher success rate of the alighting stop estimation by applying the trip-chain-based alighting stop estimation methodology in the first stage and the machine learning-based methodology in the second stage [25].

\subsection{Implication}

To date, various methodologies have been developed and applied to estimate alighting stops, including the trip-chain-based estimation methodology and AI/probability model-based estimation methodology. Research has been continued to validate the effectiveness and accuracy of the methods and improve the methodologies to facilitate more accurate alighting stop estimation for more trips. However, when a single methodology is applied, there is the limitation that the scope of the applicable trips is limited.

In this study, we propose a methodology for estimating the alighting stop by stage by considering the characteristics and limitations of the trip-chain-based alighting stop estimation methodology and the past history data-based alighting stop estimation methodology. This study aims to build more accurate and complete data for the travel patterns of the public transport users for monitoring and decision-support of public transport operations. To this end, we classify trip types by considering the continuity and recurrence of trips based on the trip history data and apply an appropriate alighting stop estimation method for each type. 
Table 1. Accuracy of destination inference results from previous studies.

\begin{tabular}{ccccc}
\hline Researcher & Method & Accuracy & Criteria for Evaluating Accuracy & Reference \\
\hline Li et al. (2011) & Trip-chain & $75 \% \sim 85 \%$ & Accuracy of OD Matrix & [6] \\
\hline Ma (2013) & Trip-chain & $91.3 \% \sim 94.6 \%$ & Within 2 stops away from actual destination & [8] \\
\hline Munizaga et al. (2014) & Trip-chain & $84.2 \%$ & Within 400m from actual destination & [20] \\
\hline He et al. (2015) & $\begin{array}{c}\text { Historical trip data } \\
\text { Analysis }\end{array}$ & $79.2 \%$ & Within $800 \mathrm{~m}$ from actual destination & [17] \\
\hline Alsger et al. (2016) & Trip-chain & $86.6 \%$ & Inferred and actual destinations located in same zone & [5] \\
\hline Shin et al. (2016) & Trip-chain & $82.4 \%$ & Within 2 stops away from actual destination & [10] \\
\hline Kim and Lee (2017) & Trip-chain & $93.6 \% \sim 94.0 \%$ & Within 2 stops away from actual destination & [13] \\
\hline Kim et al. (2018) & Trip-chain & $90.5 \%$ & Within 2 stops away from actual destination & [26] \\
\hline Yoo et al. (2019) & $\begin{array}{c}\text { Trip-chain and historical trip } \\
\text { data analysis }\end{array}$ & $67.2 \%$ & Within 1 stop away from actual destination & [19] \\
\hline Lee (2019) & $\begin{array}{c}\text { Trip-chain and Gaussian } \\
\text { Mixture Model }\end{array}$ & $86.1 \%$ & $\begin{array}{c}\text { Inferred and actual destinations located in same zone } \\
\text { [25] }\end{array}$ \\
\hline Yan et al. (2019) & $\begin{array}{c}\text { Trip-chain and machine } \\
\text { learning }\end{array}$ & $74.43 \%$ & Within 2 stops away from actual destination & [22] \\
\hline Shin (2020) & Spatial clustering & $80.0 \%$ & Within 400m from actual destination
\end{tabular}

Note: The accuracy of trip destination inference refers to the percentage of trips where the inferred destination matches the actual destination.

\section{Data}

The data used in this study contain bus and subway boarding and alighting information and consist of card ID, boarding stop and time, alighting stop and time, used route, etc. We used one-year transportation card data of 2020 for the whole country to estimate the transportation card-based alighting stop operation information (alighting stop, alighting time) considering the characteristics of the trip types. However, when a passenger boarded a bus and then used a subway on the next trip, we fabricated the data using the location coordinates of the stop to construct the trip-chain. The total number of data (based on buses) used in the analysis was 4,110,205,036 (approximately 4.1 billion/month), out of which about $17 \%$ had no alighting tag information. However, when we examine for each city or province, the percentage of the missing alighting tag data in the remaining regions after excluding the Seoul metropolitan area and Daejeon \& Sejong areas is about $63 \%$, which presents a limitation in using the data for the analysis of real travel patterns in the public transportation. Therefore, alighting stop estimation is imperative.

Table 2. Result of analysis on the status of alighting non-tag by city and province based on traffic card data.

\begin{tabular}{cccc}
\hline region & total & Alighting non-tag data & ratio \\
\hline Seoul & $136,502,534$ & $3,933,416$ & $2.90 \%$ \\
\hline Busan & $37,366,459$ & $21,420,213$ & $57.30 \%$ \\
\hline Incheon & $25,191,574$ & 780,977 & $3.10 \%$ \\
\hline Gwangju & $8,365,264$ & $5,823,725$ & $69.60 \%$ \\
\hline Daejeon & $10,800,434$ & $1,691,237$ & $15.70 \%$ \\
\hline Ulsan & $6,753,704$ & $4,767,535$ & $70.60 \%$ \\
\hline Sejong & $1,590,931$ & 154,489 & $9.70 \%$ \\
\hline Gyeonggi-do & $124,531,413$ & $1,588,267$ & $1.30 \%$ \\
\hline Gangwon-do & $3,247,020$ & $63.40 \%$ \\
\hline Chungcheongbuk-do & $4,317,692$ & $57.40 \%$ \\
\hline Chungcheongnam-do & $4,695,978$ & $2,478,044$ & $35.30 \%$ \\
\hline Jeollabuk-do & $4,700,207$ & $1,658,766$ & $73.50 \%$ \\
\hline Jeollanam-do & $3,916,794$ & $3,455,147$ & $73.10 \%$ \\
\hline Gyeongsangbuk-do & $4,823,337$ & $80.90 \%$ \\
\hline Gyeongsangnam-do & $13,049,527$ & $3,863,507$ & $76.60 \%$ \\
\hline Jeju & $4,248,946$ & $3,902,572$ & $3.80 \%$ \\
\hline total & $394,101,814$ & $9,990,513$ & $17.25 \%$ \\
\hline
\end{tabular}

\section{Methodology}

\subsection{Definition of trip type}


In this study, a trip is defined as from the time of boarding a bus to the time of alighting the bus. Furthermore, we check whether the alighting stop information is missing for individual trips, and in the case of trips with missing alighting tag information, we classify the trip type in terms of the trip continuity and recurrence based on the identifiable information of other trips of the pertinent card ID. For this, it is presumed that the same card ID is maintained for the entire time range of the utilized data.

To determine the trip type, we built the basic DB using the boarding and alighting information in the sequential order of time for all trips on a pertinent day and the first trip on the next day for each ID. In the case of the first trip on the next day, the data were constructed for the estimation in the event that the passenger did not tag the card when alighting the bus on a pertinent day.

The trip-chain-based alighting stop estimation methodology assumes that the passenger boards a bus around the position where the $i$ route, $j$-th alighting stop information is missing. In other words, a trip must occur on the same day or the next day after the alighting tag information is missing, and an estimation-target route must pass through the boarding stop of the next trip within the allowed walking distance $\left(d_{l}=500 \mathrm{~m}\right)$. If all of these conditions are met, the trip is considered to have occurred continuously and classified as a "continuous travel"; otherwise, it is classified as a "non-continuous travel".

The concept of continuous travel is different from that of transfer. If a passenger used two bus routes by transferring once in the middle during the process of traveling from the origin to the destination, the trip is continuous travel. Furthermore, if the passenger travels from A to B without transferring and then starts the next trip at B or in the vicinity (within the allowed walking distance) on the same day or the next day, the trip is also defined as continuous travel.

In the case of non-continuous travel, because the alighting stop is estimated by searching the past travel history and finding a trip that is determined to have the similarity temporally and spatially to the trip with missing alighting tag, it is selected by using the card ID's past travel history DB. A trip with temporal similarity refers to a case of boarding a certain number of times (three times per week) or more at the same stop at a similar time, and since the trip occurs repeatedly, it is classified as "recurrent travel". For example, if a passenger uses a bus to go to work from A to B (trip 1), uses a means other than public transportation to go home from B to A after work (no travel record), and uses a bus to go to work from A to B on the next day (trip 2), then trip 1 and trip 2 are non-continuous travels. However, if the trip for commuting from A to B repeats more than a certain number of times, it is classified as "recurrent travel".

A trip without even the temporal similarity is classified as "non-continuous", "nonrecurrent" travel, and the alighting stop is estimated based on the spatial similarity. In this study, if there is no past travel history of the card ID for the analyzed period, we use the boarding and alighting patterns between the stops of the route to estimate the alighting stop.

\subsection{Method}

In this study, we estimate the missing alighting stop by applying an appropriate method for each type of travel with missing alighting stop information, which was classified above. First, in the case of "continuous travel", the trip-chain-based alighting stop estimation method is applied, which estimates the missing alighting stop based on the boarding stop information of the next trip. Second, for the "recurrent travel" among "noncontinuous travels", we analyze the past travel history information to check the travel pattern for each card ID and then analyze the potential residence/workplace, based on which the missing alighting stop is estimated. Third, for the "non-recurrent travel", we extract the history of boarding around the boarding stop of the estimation-target trip from the past travel history information and estimate the alighting stop based on the destination information in the past travel history. Finally, if there is no continuous travel or past 
travel history, the alighting stop is assigned probabilistically based on the origin-destination (OD) pattern for each route. Table 2 shows the utilized data for each stage.

Table 2. Utilization data for estimating destination of bus trip by travel type.

\begin{tabular}{ccccc}
\hline travel type & 1. Continuous travel & $\begin{array}{c}\text { 2. non-continuous } \\
\text { recurrent travel }\end{array}$ & $\begin{array}{c}\text { 3. non-continuous } \\
\text { non-recurrent travel }\end{array}$ & 4. route pattern \\
\hline input data & The day and tommorow (per & $\begin{array}{c}\text { Individual potential } \\
\text { residence/workplace data } \\
\text { (per month) }\end{array}$ & $\begin{array}{c}\text { Individual OD travel pattern Route OD travel pattern data } \\
\text { data (per year) }\end{array}$ & (per year) \\
\hline Data for validation & & April 2020 (one month)/ Verification by day of the week & \\
\hline
\end{tabular}

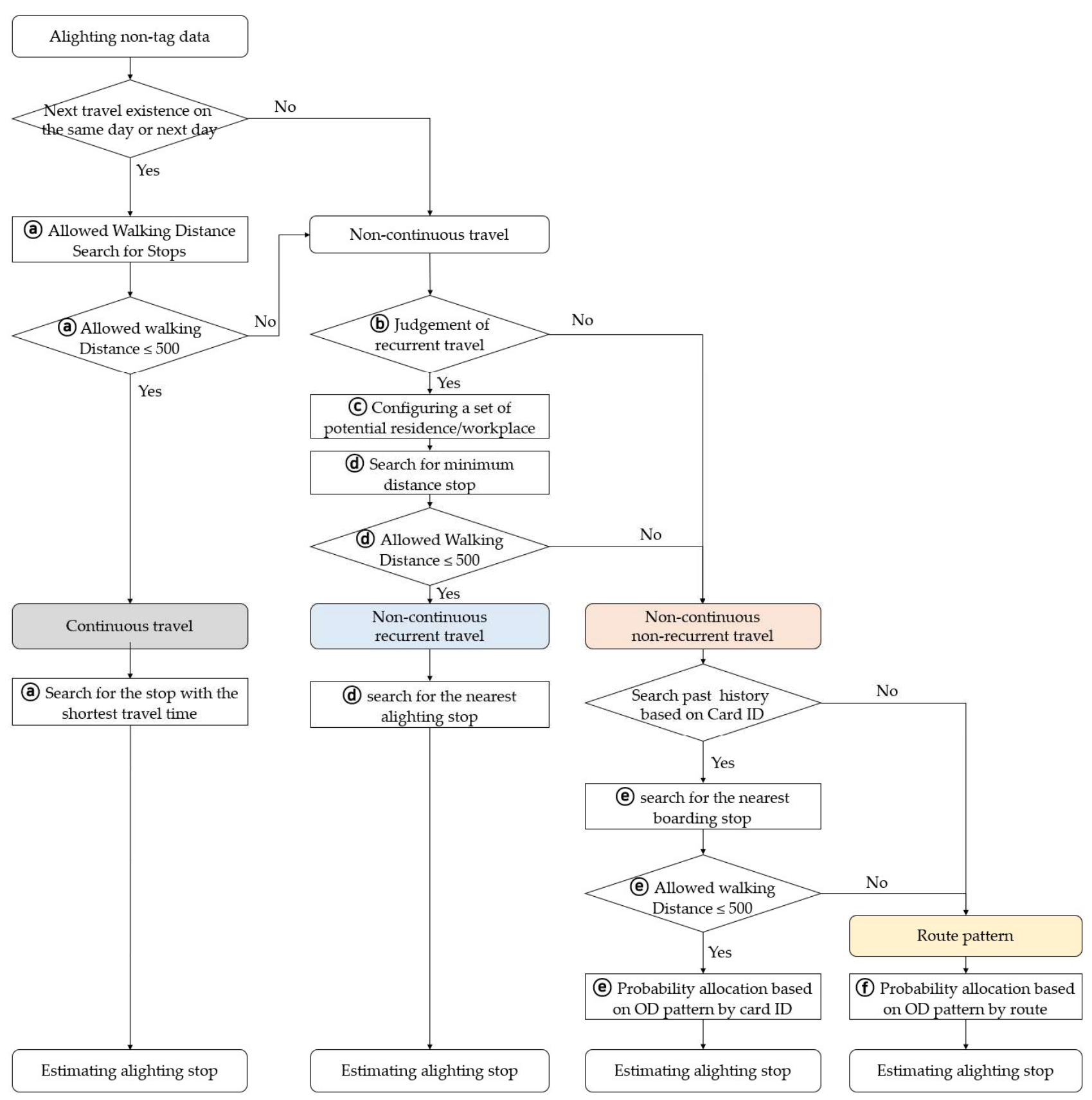

Figure 1. Method of step-by-step estimating destination of bus trip considering trip type characteristics: (a) Judgment of continuous travel, (b) Judgment of recurrent travel, (c) Configuring a set of potential residence/workplace, (d) Non-continuous recurrent travel, (e) Non-continuous non-recurrent travel, $f$ Route pattern. 
The alighting-stop estimation method proposed in this study performs the estimation according to the stage, as shown in Fig. 1, and if the estimation is impossible in each stage, it proceeds to the next stage. Furthermore, the results estimated through Stages 1 and 2 (continuous travel, non-continuous \& non-recurrent travel) are used as input data for Stages 3 and 4 . The alighting stop estimation method in each stage is as follows.

Stage 1: The missing alighting stop information for "continuous travel" is estimated by applying the trip-chain-based method. It is assumed that the passenger alighted the bus around the boarding stop of the immediate next trip (on the same day or the next day) of the trip that has the missing alighting information. Here, we also considered the subway station when the continuous data after the missing data of alighting the bus is pertaining to a subway. To estimate the alighting stop for continuous travel, we estimate the traveling time (TT) between the stops $(i)$ located after the boarding stop on the route of the alighting information-missing trip and the boarding stop $(j)$ of the next trip. As shown in Eq. (1), TT is defined as the sum of the travel time $\left(t t_{i}\right)$ from the boarding stop to the $i$-th stop and the walking time $\left(w t_{i, j}\right)$ from the $i$-th stop to the $j$-th stop. $w t_{i, j}$ is calculated by dividing the distance between the stops by the average walking speed $(\mathrm{ws}=4 \mathrm{~km} / \mathrm{h}$ ), as shown in Eq. (2). For the calculation of the distance $(d)$ between the stops, we use the Euclid distance, which is calculated based on the latitude coordinate $\left(P_{x}\right)$ and longitudinal coordinate $\left(P_{y}\right)$ data of the stops. Here, the distance between the stops $i$ and $j$ is calculated considering the bus-stop stopping order and direction for each route. In other words, a stop that minimizes the travel time is selected as the alighting stop, and here, we target the stops within the allowed walking distance $\left(d_{s t}=500 \mathrm{~m}\right)$ for the distance between the stops.

$$
\begin{gathered}
T T_{i}=t t_{i}+w t_{i, j}, \quad \forall i \\
w t_{i, j}=\frac{\sqrt{\left(P_{j, x}-P_{i, x}\right)^{2}+\left(P_{j, y}-P_{i, y}\right)^{2}}}{w s} \quad \forall i \\
\left.S_{i}=\min T T_{i}, \quad \text { s.t. } d<d_{l} \text { (assumed } 500 m\right)
\end{gathered}
$$

(1) Extraction of passenger boarding stop information
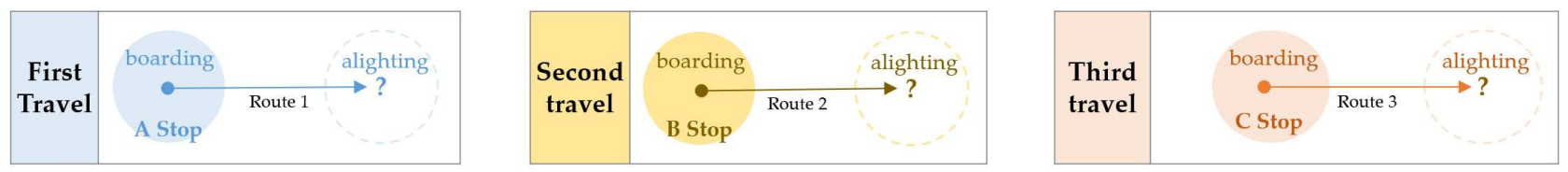

(2) Estimation of the alighting stop based on the next travel boarding stop

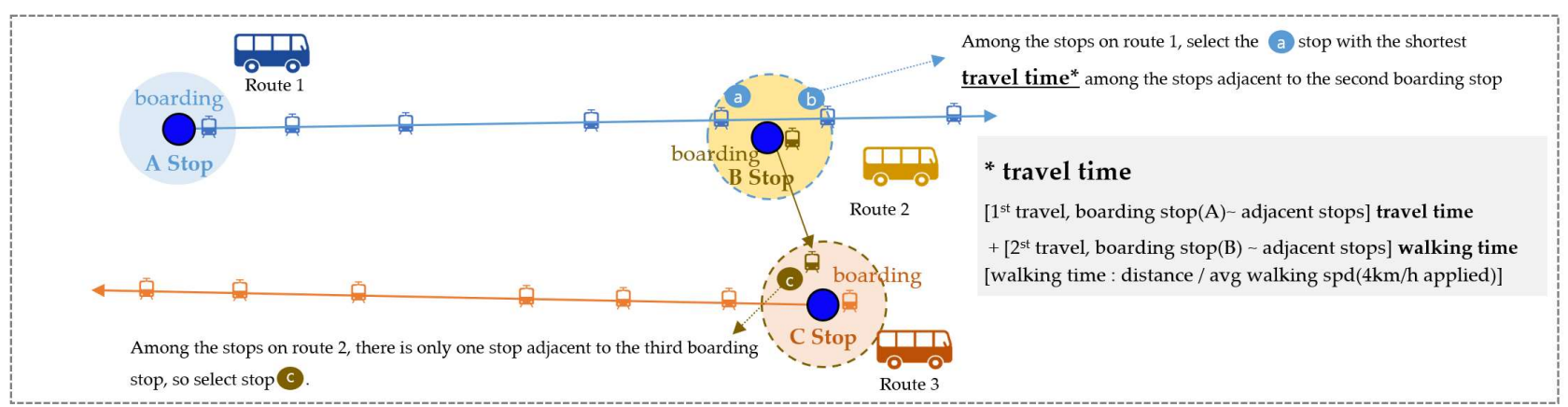

Figure 1. Method of estimating the alighting stop for continuous travel. 
Stage 2: To estimate the alighting stop for a "recurrent travel" among the "non-continuous travels", analysis is performed first for the past travel history of each person. Recurrent travels are mainly commuting trips of moving from the residence to the workplace and from the workplace to the residence. Potential workplace and residence are selected by using only the boarding information for each card ID to build the recurrent pattern data. As for the selection criteria, we count the number of boarding at each boarding stop for each card ID during the morning commute hours (04:00-12:00) and the afternoon commute hours (15:00-02:00). A stop that satisfies the criteria of a certain boarding frequency (three or more per week) among the boarding stops in the morning commute hours is selected as a "potential residence"; based on the same method, the boarding stop in the afternoon commute hours is selected as a "potential workplace". As for the criteria for determining recurrent travels, we selected three times or more per week by referencing a case of mobile data-based staying purpose classification (Song and Lee, 2018), which is similar in the aspect of determining travel purposes and public transportation data. Here, we also included the potential stop candidates and stops existing within $500 \mathrm{~m}$, among the stops used less than three times per week. This is to include the cases of commuting using other routes nearby.

This task is to examine the stops that have recurrent patterns based on the boarding stop information, and it is not to determine the residence and workplace accurately. In the alighting stop estimation based on the travel patterns of each person, the use time of the alighting stop information-missing trip is checked; the distance $(d)$ is calculated between the stops located on the used route of the alighting information-missing trip and the stop at the "potential residence or workplace candidates"; a stop that minimizes the distance while locating with the allowed walking distance $\left(d_{s t}\right)$ is estimated to be the alighting stop. Here, in the case of trips during the morning commute hours, stops are searched around the stop at the potential workplace, and in the case of the afternoon commute hours, stops are searched around the stop at the potential residence.

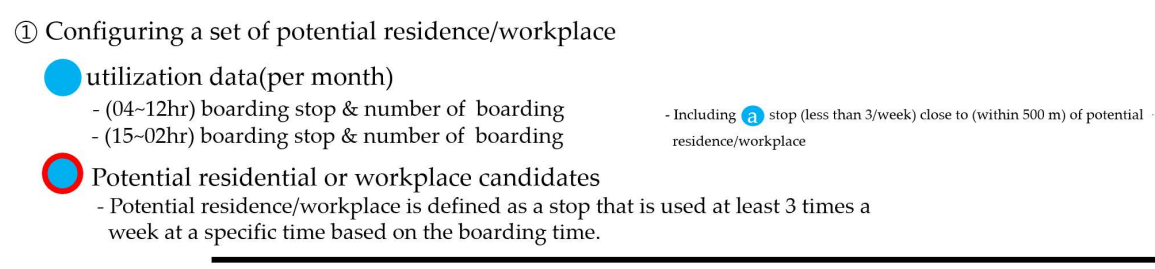

(2) Estimation of the alighting stop based on a set of potential residence/workplace

Estimated alighting stop

- If there is a potential residence/work stop candidate among the stops on the boarding route or is adjacent, it is estimated as an alighting stop.

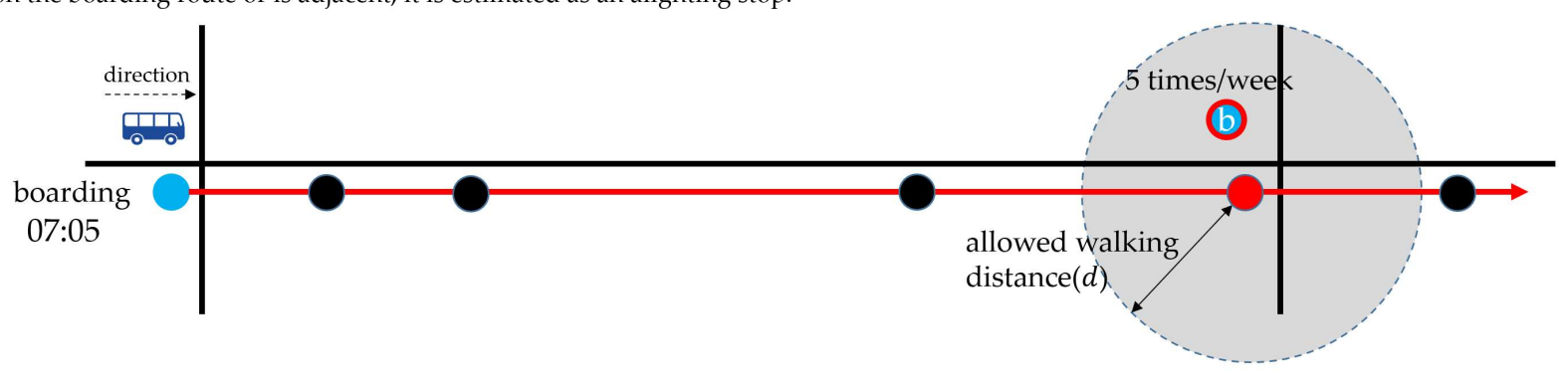

Figure 2. Method of estimating the alighting stop for non-continuous recurrent travel.

Stage 3: In the case of "non-recurrent travel" among "non-continuous travels", the alighting stop is estimated by searching the past travel history and finding the trip that seems to be similar to the alighting information-missing trip. As for the travel history of 
each card ID, we build an alighting stop set for each boarding stop of non-recurrent trips (less than three times per week) by using the data that contain both boarding and alighting information. The non-recurrent travel pattern data are built by classifying the weekdays and weekends since the difference in the patterns is expected to be large between each day of the week. Based on the OD pattern data for each card ID, we use the percentage of each alighting stop for each boarding stop to estimate the alighting stop. Here, if the boarding stop is not found in the pattern data, we search stops within a short distance (within $500 \mathrm{~m}$ ) to determine whether the pattern data exist or not, and if there is another route, the corresponding pattern data are used to estimate the alighting stop based on the same method. If there is no pattern data, we proceed to the next stage.

Stage 4: In the case of "route pattern", if it is impossible to estimate the alighting stop in Stages 1 through 3, we estimate it based on the OD pattern (one-year basis) for each route. This is to build $100 \%$ valid data for public transportation status analysis and utilization.

\section{Results}

\subsection{Analysis overview}

We conducted an analysis using the transportation card data of the Seoul metropoli$\tan$ area, in which there is almost no missing alighting tag information, to apply the proposed trip type classification and alighting stop estimation methods and examine the accuracy. The time range of the utilized data was one year in 2020. For the validation of the alighting stop estimation methodology developed in this study, we used one-month (April 202) data of the Seoul metropolitan sphere (Seoul, Gyeonggi Province, and Incheon) where the non-tagged alighting ratio was low. Since the non-tagged alighting ratio is very low in the case of subways, we selected only the bus use data as the estimation target. First, we built complete data (data with complete boarding-alighting records) for the accuracy validation of the alighting stop estimation method and deleted all alighting information from the data. Then, we tested the model's accuracy and identified the valid data ratio through the alighting stop estimation in each stage, considering the trip type based on the data. Furthermore, since the travel patterns may be significantly different between each day of the week, we also performed the validation for each day of the week.

\subsection{Classification of trip types}

In this study, we classified four types (continuous, non-continuous and recurrent, non-continuous and non-recurrent, and route pattern) for the bus trips in April 2020 $(210,920,089$ trips/month) in the Seoul metropolitan sphere. Continuous travel refers to a case where a trip is recorded on the same day or the next day after taking a bus trip of the classification target. To determine the spatial continuity, we classified a trip as continuous travel, only when there was a stop on the used route of the classification-target trip within a certain distance from the boarding stop of the next trip. According to the result of sensitivity analysis on allowed walking distance in a previous study, the change in the success rate and accuracy of the estimation is not large when it is around $500 \mathrm{~m}$ (Kim and Lee, 2017). In some cases, the allowed walking distance is set to $500 \mathrm{~m}$, considering that the common radius of a subway station area is $500 \mathrm{~m}$, or it is set to $500-1,000 \mathrm{~m}$ based on the distance distribution between the bus stops in the analyzed area (Alsger et al., 2016, Nunes et al., 2016, Gordon et al., 2013, Yang et al., 2019). In this study, we set the allowed walking distance to $500 \mathrm{~m}$, considering the above previous studies. According to the analysis results, about $67.68 \%$ of the bus trips are classified as continuous travels.

Non-continuous travels are classified into recurrent travels and non-recurrent travels. To determine the recurrence of the trips, we analyzed one-month boarding data and counted the boarding frequency for each boarding stop in each time range for each card ID. To determine the residence and workplace of a recurrent travel, we established the "morning commute hours" and "afternoon commute hours". The time range was set broadly to reflect various commute patterns: 04:00-12:00 for the morning commute hours 
and from 15:00 to 02:00 on the next day for the afternoon commute hours. Moreover, we selected the stop where the passenger boarded three times or more per week on average in each time range as the stop at a residence or workplace candidate. The boarding stops between 04:00 and 12:00 are classified as residence candidates, and the boarding stops between 15:00 and 02:00 on the next day are classified as workplace candidates. As a result of classifying the trip type, $13,377,146(6.34 \%)$ trips were counted as non-continuous recurrent travels. When no candidate stops of residence and workplace were selected, the non-continuous travels were classified as non-recurrent travels (19.80\%). We used the pattern data (weekdays/weekends) built based on the one-year boarding/alighting OD data for each card ID.

In the case of route patterns, the one-year OD patterns for each route ID were constructed for all trips that could not be estimated as continuous/non-continuous trips, and the alighting stop percentages were calculated based on the boarding stop to assign the alighting stop based on the percentage. Here, we considered the moving direction on the route and assigned the alighting stop based on the probability of the corresponding stop after the route stop. As a result of the trip type classification, 13,038,870 (6.18\%) trips were counted as route patterns.

\begin{tabular}{|c|c|c|c|}
\hline \multicolumn{2}{|c|}{ Table 2020. Division. } & Number of data & Ratio \\
\hline \multicolumn{2}{|c|}{ Continuous travel } & $142,743,224$ & $67.68 \%$ \\
\hline \multirow{2}{*}{ non-continuous } & recurrent travel & $13,377,146$ & $6.34 \%$ \\
\hline & non-recurrent travel & $41,760,849$ & $19.80 \%$ \\
\hline \multicolumn{2}{|c|}{ route pattern } & $13,038,870$ & $6.18 \%$ \\
\hline \multicolumn{2}{|c|}{ Total } & $210,920,089$ & $100.00 \%$ \\
\hline
\end{tabular}

\subsection{Estimated results of destination by trip type characteristics}

We calculated the estimation rate and accuracy for each trip type to validate the effectiveness of the methodology proposed in this study. The estimation rate refers to the percentage of the trips for which we can estimate the alighting stops satisfying the alighting stop estimation criteria for each stage. For the accuracy, we analyzed whether the estimated alighting stops were the same as the actual alighting stops, respectively, or comparatively analyzed the results according to the straight distance $(0-1,500 \mathrm{~m})$ between the estimated and actual alighting stops. Lastly, we performed the validation for each day of the week to determine whether the travel patterns in each day of the week (weekdays/weekends) were appropriately reflected in the estimation.

Under the assumption that the alighting tag data of the public transportation cards were $100 \%$ missing, the analysis showed that $49.66 \%$ of the estimated stops were the same as the actual stops, and in the case of less than $1 \mathrm{~km}$, the destination could be identified for $85.00 \%$. Upon analyzing the accuracy in each stage based on a range of within $1 \mathrm{~km}$, we found that the accuracy was $94.39 \%$ for the continuous travels, $81.55 \%$ for the noncontinuous, non-recurrent travels, $66.53 \%$ for the non-continuous, non-recurrent travels, and $44.92 \%$ for the route patterns. The assumption of the trip-chain-based alighting stop estimation method for continuous travels, i.e., the assumption that the passenger begins the next trip around the destination of the previous trip is valid. Furthermore, it is effective to apply the trip-chain-based alighting stop estimation methods to Stage 1.

Non-continuous, recurrent travel can be seen as a case where despite repeatedly traveling between certain origin and destination, the user does not travel every day or uses a means other than public transportation due to a special circumstance, resulting in a disconnection between the trips. Although the percentage of the non-continuous, recurrent travels in the total bus trips is $6.34 \%$, which is not high, this trip type can be used as a supplementary method to estimate the alighting stops more effectively for disconnected trips through regularity. Furthermore, since this trip type shows better results when it is compared to the non-recurrent travels in terms of accuracy, it is appropriate to apply it to Stage 2.

For the non-continuous, non-recurrent travels, the missing alighting stops are estimated based on the past travel history (weekdays and weekends are distinguished) for 
each card ID. Based on the fact that the passenger's residence and major destination do not change much, even if the passenger does not travel to the destination repeatedly according to the travel history of each person, it is confirmed that this trip type can be used to supplement the alighting stop estimation method. However, when the error between the estimated and actual alighting stops is less than $1 \mathrm{~km}$, the accuracy is $66.53 \%$, which is somewhat low compared to that of the recurrent travels. Furthermore, among the noncontinuous, non-recurrent travels, $6.18 \%$ accounted for the case where the alighting stops could not be estimated, and the estimation is impossible if there is no past travel history.

Finally, the route pattern-applied alighting stop estimation is performed when the estimation is impossible in Stages 1 through 3 performed earlier. According to the alighting stop estimation results, $12.32 \%$ of the estimated stops are the same as the actual alighting stops, and $44.92 \%$ accounts for the case where the distance (error) between the estimated and actual alighting stops is less than $1 \mathrm{~km}$. The accuracy is relatively low because route patterns, not each person's patterns, are applied.

Upon examining the accuracy (less than $1 \mathrm{~km}$ ) of the alighting estimation for each stage, we found that the accuracy for the continuous travels was the highest $(94.39 \%)$, and that of the results using route patterns was the lowest (44.92\%). This study aims to ensure that more complete information is reflected when monitoring operations and making decisions by building a public transportation dataset that can be used in the field. To this end, we have designed a methodology that ensures a $100 \%$ valid tag rate by applying the route patterns in the last stage. Although the accuracy is somewhat low in the case of route patterns, there will be no problem in using them in the actual field because the composition ratio is relatively low at $6.18 \%$.

Table 2. Estimated results of destination by trip type characteristics.

\begin{tabular}{|c|c|c|c|c|c|c|}
\hline & Division & $\begin{array}{c}\text { Continuous } \\
\text { travel }\end{array}$ & $\begin{array}{l}\text { Non-continuous } \\
\text { recurrent travel }\end{array}$ & $\begin{array}{c}\text { Non-continuous } \\
\text { non-recurrent travel }\end{array}$ & $\begin{array}{c}\text { Route } \\
\text { pattern }\end{array}$ & Total \\
\hline & Number of data & $142,743,224$ & $13,377,146$ & $41,760,849$ & $13,038,870$ & $210,920,089$ \\
\hline & Ration & $67.68 \%$ & $6.34 \%$ & $19.80 \%$ & $6.18 \%$ & $100.00 \%$ \\
\hline \multirow{4}{*}{ same } & matches & $85,533,555$ & $5,657,869$ & $11,947,068$ & $1,606,002$ & \multirow{4}{*}{$49.66 \%$} \\
\hline & accuracy & $59.92 \%$ & $42.30 \%$ & $28.61 \%$ & $12.32 \%$ & \\
\hline & matches (accumulate) & $85,533,555$ & $91,191,424$ & $103,138,492$ & $104,744,494$ & \\
\hline & accuracy (accumulate) & $59.92 \%$ & $58.41 \%$ & $52.12 \%$ & $49.66 \%$ & \\
\hline \multirow{4}{*}{$500 \mathrm{~m}$} & matches & $120,740,211$ & $9,325,434$ & $21,519,778$ & $3,576,205$ & \multirow{4}{*}{$73.56 \%$} \\
\hline & accuracy & $84.59 \%$ & $69.71 \%$ & $51.53 \%$ & $27.43 \%$ & \\
\hline & matches (accumulate) & $120,740,211$ & $130,065,645$ & $151,585,423$ & $155,161,628$ & \\
\hline & accuracy (accumulate) & $84.59 \%$ & $83.31 \%$ & $76.60 \%$ & $73.56 \%$ & \\
\hline \multirow{4}{*}{$1000 \mathrm{~m}$} & matches & $134,729,489$ & $10,909,099$ & $27,783,953$ & $5,856,696$ & \multirow{4}{*}{$85.00 \%$} \\
\hline & accuracy & $94.39 \%$ & $81.55 \%$ & $66.53 \%$ & $44.92 \%$ & \\
\hline & matches (accumulate) & $134,729,489$ & $145,638,588$ & $173,422,541$ & $179,279,237$ & \\
\hline & accuracy (accumulate) & $94.39 \%$ & $93.29 \%$ & $87.64 \%$ & $85.00 \%$ & \\
\hline \multirow{4}{*}{$1500 \mathrm{~m}$} & matches & $137,472,068$ & $11,488,371$ & $31,148,767$ & $7,449,592$ & \multirow{4}{*}{$88.92 \%$} \\
\hline & accuracy & $96.31 \%$ & $85.88 \%$ & $74.59 \%$ & $57.13 \%$ & \\
\hline & matches (accumulate) & $137,472,068$ & $148,960,439$ & $180,109,206$ & $187,558,798$ & \\
\hline & accuracy (accumulate) & $96.31 \%$ & $95.41 \%$ & $91.02 \%$ & $88.92 \%$ & \\
\hline
\end{tabular}

According to the analysis on the alighting stop estimation results for each day of the week, the accuracy is low in the case of weekends (Saturday/Sunday) than in the case of weekdays. When the accuracy is examined for each stage, we find that there is no significant difference between the weekdays and the weekends in the cases of continuous travels and non-continuous, recurrent travels. However, in the cases of the non-continuous, nonrecurrent travels and the route patterns, the accuracy is $2.5-5.0 \%$ lower on the weekends compared to the weekdays. The accuracy of the pattern-based estimation method is relatively low because the variability of the weekend trip patterns is relatively high. 


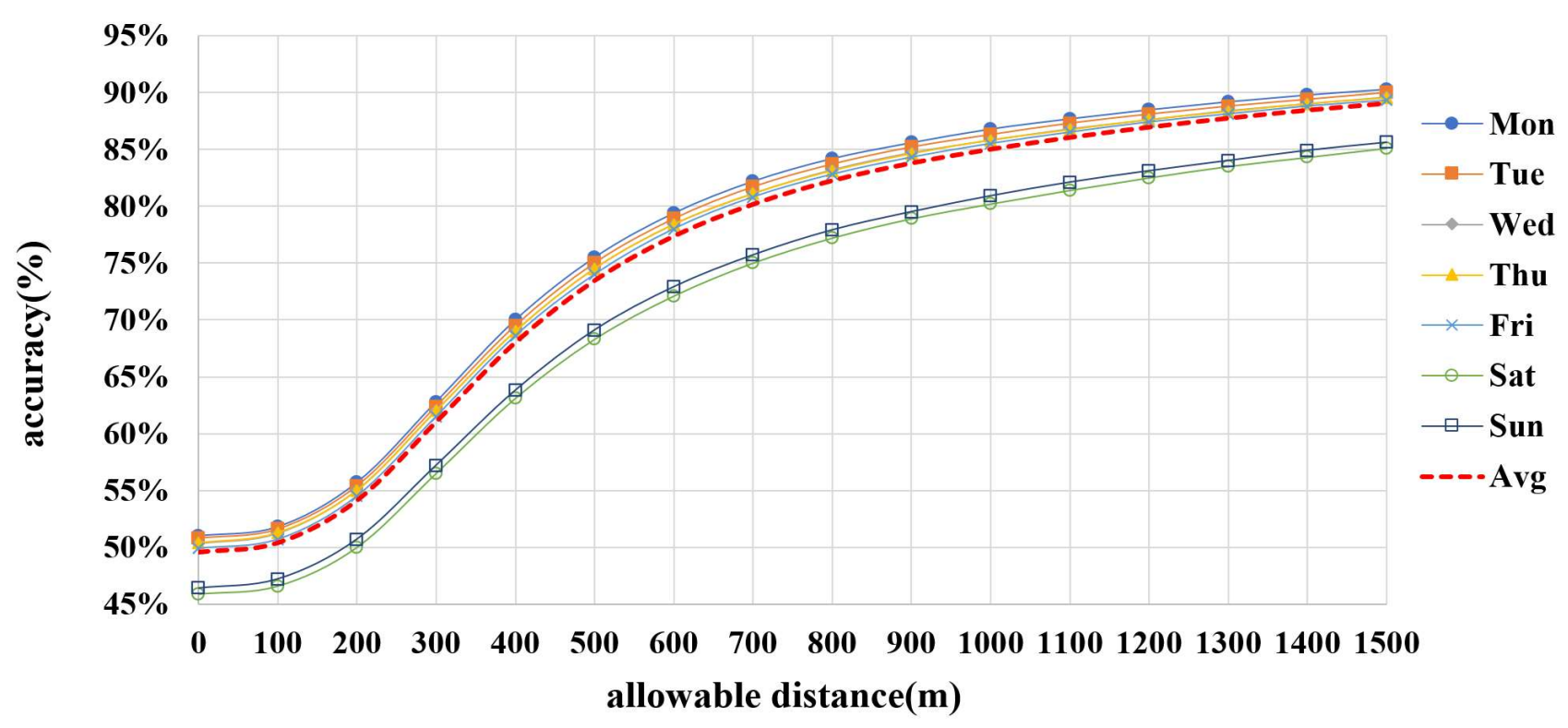

Figure 3. Result of destination estimation accuracy.

\section{Conclusions}

This study aimed to develop a methodology for estimating non-tagged alighting stop information stage-by-stage by considering the characteristics of trip types and using transportation card data. We classified four trip types based on the continuity and recurrence of trips and developed the methodology with a trip-chain-based estimation method that considers the characteristics of individual trip type, an estimation method based on the recurrent travel patterns and non-recurrent travel patterns for each person (card ID), and finally, route patterns for building $100 \%$ valid data. The methodology proposed in this study was applied stage-by-stage to transport card data of the Seoul metropolitan area, and the estimation accuracy was examined. The results of trip type classification show that in about $67.68 \%$ of the cases, travel information exists on the same day as the alighting information-missing trip or the next day while the trip is determined to be temporally and spatially continuous from the previous trip. Therefore, it is valid to apply the trip-chainbased alighting stop estimation methodology first. According to the alighting stop estimation results, the accuracy (less than $1 \mathrm{~km}$ ) of the alighting stop estimation for all samples is about $85.00 \%$ based on a scenario where the alighting tag data in bus trips are $100 \%$ missing, and the usable valid tag rate is close to $100 \%$. In short, the alighting stop estimation's accuracy and valid tag rate show significant improvements compared to those of previous studies.

In some trips, the estimation of alighting stops is impossible based on only a single methodology, such as the trip-chain-based estimation methodology and pattern-based estimation methodology. In this study, we classified the trip types by analyzing the characteristics of transportation card data to build $100 \%$ complete data. Furthermore, this study differs from previous studies in that it built more complete bus trip data by applying the stage-by-stage alighting estimation model. In the future, we need to conduct additional studies to apply and review more various estimation methods such as AI-based alighting stop estimation methodology to improve the alighting estimation accuracy. Addition research is also required to improve the accuracy of alighting estimation for weekend trips. This should lead to an improvement of the current public transport OD methodology and the discovery of public transportation policies and services.

Author Contributions: Conceptualization, S.L., S.C., and J.L.; methodology, S.L., S.C., and J.L.; formal analysis, S.L., S.C., and J.L.; validation, S.L., B.B., and D.N.; data curation, S.L., S.C., and J.L.; 
writing-original draft preparation, S.L., B.B., and D.N.; writing-review and editing, S.L., B.B., and D.N.; supervision, B.B., D.N., and S.C. All authors have read and agreed to the published version of the manuscript.

Funding: This paper is supported by the Korea Agency for Infrastructure Technology Advancement(KAIA) grant funded by the Ministry of Land, Infrastructure and Transport (Grant 21TLRPB148671-04). This paper is supported by Inha University

Institutional Review Board Statement: Not applicable.

Data Availability: The data presented in this study are available on request from the corresponding author. The data are not publicly available due to privacy.

Conflicts of Interest: The authors declare no conflict of interest.

\section{References}

1. Li, T.; Sun, D.; Jing, P.; Yang, K. Smart card data mining of public transport destination: A literature review, information, $2018,9(1), 1-21$.

2. Hussain, E.; Bhaskar, A.; Chung, E. Transit OD matrix estimation using smartcard data: Recent developments and future research challenges, Transportation Research Part C: Emerging Technologies, 2021, 125, 103044.

3. Barry, J. J.; Newhouser, R.; Rahbee, A.; Sayeda, S. Origin and destination estimation in New York City with automated fare system data, Transportation Research Record, 2002, 1817(1), 183-187.

4. Munizaga, M. A.; Palma, C. Estimation of a disaggregate multimodal public transport Origin-Destination matrix from passive smartcard data from Santiago, Chile, Transportation Research Part C: Emerging Technologies, 2012, 24, 9-18.

5. Shin, K.W. Inferring the Transit Trip Destination Zone of Smart Card User Using Trip Chain Structure, J. Korean Soc. Transp. 2016, 34(5), 437-448.

6. Li, D.; Lin, Y.; Zhao, X.; Song, H.; Zou, N. Estimating a transit passenger trip origin-destination matrix using automatic fare collection system, International Conference on Database Systems for Advanced Applications, 2011, 502-513.

7. Wang, W.; Attanucci, J. P.; Wilson, N. H. Bus passenger origin-destination estimation and related analyses using automated data collection systems, Journal of Public Transportation, 2011, 14(4), 131-150.

8. Xiaolei Ma. Smart card data mining and inference for transit system optimization and performance improvement, Ph. D. diss., University of Washington, 2013.

9. Nunes, A. A.; Dias, T. G.; Cunha, J. F. Passenger journey destination estimation from automated fare collection system data using spatial validation, IEEE transactions on intelligent transportation systems, 2015, 17(1), 133-142.

10. Kim, K.T.; Lee, I.M. Public Transportation Alighting Estimation Method Using Smart Card Data, Journal of the Korean society for railway. 2017, 20(5), 692-702.

11. Alsger, A. A.; Mesbah, M.; Ferreira, L.; Safi, H. Use of smart card fare data to estimate public transport origin-destination matrix, Transportation Research Record, 2015, 2535(1), 88-96.

12. Trepanier, M.; Chapleau, R. Destination estimation from public transport smartcard data, IFAC Proceedings, 2006, 39(3), 393-398.

13. Kim, K.T.; Lee, I.M.; Min, J.H. Bus OD Matrix Estimation Using Smart Card Data, Journal of the Korean society for railway. 2018, 21(11), $1155-1161$.

14. Zhao, J.; Rahbee, A.; Wilson, N. H. Estimating a rail passenger trip origin-destination matrix using automatic data collection systems, Computer-Aided Civil and Infrastructure Engineering, 2007, 22(5), 376-387.

15. Barry, J. J.; Freimer, R.; Slavin, H. Use of entry-only automatic fare collection data to estimate linked transit trips in New York City, Transportation research record, 2009, 2112(1), 53-61.

16. Munizaga, M.; Devillaine, F.; Navarrete, C.; Silva, D. Validating travel behavior estimated from smartcard data, Transportation Research Part C: Emerging Technologies, 2014, 44, 70-79.

17. Alsger, A.; Assemi, B.; Mesbah, M.; Ferreira, L. Validating and improving public transport origin-destination estimation algorithm using smart card fare data, Transportation Research Part C: Emerging Technologies, 2016, 68, 490-506.

18. Nassir, N.; Khani, A.; Lee, S. G.; Noh, H.; Hickman, M. Transit stop-level origin-destination estimation through use of transit schedule and automated data collection system, Transportation Research Record, 2011, 2263(1), 140-150.

19. Lee, I.M. Estimation of Bus-Trip Destinations Using Temporal Travel Patterns of Smart Card Data, Ph. D. diss., Seoul national University, 2019.

20. He, L.; Nassir, N.; Trépanier, M.; Hickman, M. Validating and calibrating a destination estimation algorithm for public transport smart card fare collection systems, CIRRELT, 2015.

21. Cheng, Z.; Trépanier, M.; Sun, L. Probabilistic model for destination inference and travel pattern mining from smart card data, transportation, 2020, 1-19.

22. Shin, Y.S. Research of Sparse Dataset Analysis for Estimating Missing Alighting Information of Public Transportation Records: Spatial Density-based Clustering, Journal of Transport Research. 2020, 27(4), 19-31.

23. Jung, J.; Sohn, K. Deep-learning architecture to forecast destinations of bus passengers from entry-only smart-card data, IET Intelligent Transport Systems, 2017, 11(6), 334-339.

24. Assemi, B.; Alsger, A.; Moghaddam, M.; Hickman, M.; Mesbah, M. Improving alighting stop inference accuracy in the trip chaining method using neural networks, Public Transport, 2020, 12(1), 89-121.

25. Yan, F.; Yang, C.; Ukkusuri, S. V. Alighting stop determination using two-step algorithms in bus transit systems. Transportmetrica A: Transport Science, 2019, 15, 1522-1542.

26. Yoo, B.S.; Choo, S.H. A Study of Estimating the Alighting Stop on the Decision Tree Learning Model Using Smart Card Data, J. Korea Inst. Intell. Transp. Syst. 2019, 18(6), 11-30.

27. Gordon, J.; Koutsopoulos, H.; Wilson, N.; Attanucci, J. Automated Inference of Linked Transit Journeys in London Using Fare-Transaction and Vehicle Location Data. Transportation Research Record: Journal of the Transportation Research Board, 2013, 2343, 17-24.

28. Song, T.J.; Lee, H.S. The Promise of Mobile Phone Signaling Data for Inferring Mobility Patterns, International Journal of Highway Engineering. 2018, 20(6), 199-209. 